\title{
Ultra-processed food intake and risk of type 2 diabetes in a French cohort of middle-aged adults
}

\author{
Bernard Srour $^{1}$, Léopold K. Fezeu ${ }^{1}$, Emmanuelle Kesse-Guyot ${ }^{1}$, Benjamin Allès ${ }^{1}$, \\ Caroline Méjean ${ }^{2}$, Charlotte Debras ${ }^{1}$, Nathalie Druesne-Pecollo ${ }^{1}$, Eloi Chazelas ${ }^{1}$, \\ Mélanie Deschasaux ${ }^{1}$, Serge Hercberg ${ }^{1,3}$, Pilar Galan ${ }^{1}$, Carlos A. Monteiro ${ }^{4}$, Chantal Julia ${ }^{1,3}$ and \\ Mathilde Touvier ${ }^{1}$ \\ ${ }^{1}$ Nutritional Epidemiology Research Team (EREN) - Inserm U1153, INRA U1125, CNAM, University of Paris 13, \\ Bobigny, France, \\ ${ }^{2}$ UMR MOISA INRA, Montpellier, France, \\ ${ }^{3}$ Avicenne hospital APHP - Public health department, Bobigny, France and \\ ${ }^{4}$ University of Sao-Paulo, Sao-Paulo, Brazil
}

\begin{abstract}
Introduction: The consumption of ultra-processed foods has been increasing during the last decades, and has been previously associated with increased risks of mortality and several chronic diseases such as cancer, cardiovascular diseases, hypertension, and dyslipidemia. The objective of this study is to assess the prospective associations between consumption of ultra-processed foods and the risk of type 2 diabetes (T2D).

Methods: Overall, 104707 participants aged at least 18 years (median age 41.5 years) from the French NutriNet-Santé cohort (20092019) were included. Dietary intakes were collected using repeated 24 hour dietary records (5.7/subject in average), designed to register participants' usual consumption for 3300 different food items. These were categorized according to their degree of processing by the NOVA classification. Associations between ultra-processed food intake and risk of type 2 diabetes were assessed using multivariable Cox proportional hazard models adjusted for known risk factors (sociodemographic, anthropometric, lifestyle, medical history and nutritional factors).

Results: Ultra-processed sugary products, fruits and vegetables, and beverages were the highest contributors of the ultra-processed category (respectively 27.9, 18.5 and 15.6\%). Ultra-processed food intake was associated with a higher risk of T2D (n=821 incident cases; hazard ratio for an absolute increment of 10 in the percentage of ultra-processed foods in the diet $=1.15(95 \%$ confidence interval 1.06 to 1.25 ); $\mathrm{P}=0.0009$, median follow-up: $6.0 \mathrm{y}, 582252$ person-years). These results remained statistically significant after adjustment for other metabolic comorbidities, for several markers of the nutritional quality of the diet (red meat and sugary drinks consumption, intakes of saturated fatty acids, sodium, sugar, dietary fiber or Healthy/Western patterns derived by principal component analysis) and after a large range of sensitivity analyses.
\end{abstract}

Conclusions: In this large observational prospective study, higher consumption of ultra-processed foods in the diet was associated with a higher risk of type 2 diabetes. These results need to be confirmed in other populations and settings, and causality remains to be established. Various dimensions of processing such as nutritional composition of the final product, food additives, contact materials, and neoformed contaminants might play a role in these associations and further studies are needed to better understand their relative contribution. Meanwhile, public health authorities in several countries recently started to recommend privileging unprocessed/ minimally processed foods and limiting ultra-processed food consumption.

Study Registration: Clinicaltrials.gov NCT03335644.

\section{Conflict of Interest}

There is no conflict of interest 